\title{
A Clinical Study on Chikungunya Fever in a Multidisciplinary Hospital of Dhaka City
}

\author{
SB RABBANI $^{\mathrm{a}}$, PR SAHA $^{\mathrm{b}}, \mathrm{MI} \mathrm{HOSSAIN}^{\mathrm{c}}, \mathrm{ABEGUM}^{\mathrm{d}}$, MJ TALUKDER $^{\mathrm{e}}$
}

\section{Summary:}

Chikungunya is one of the most rapidly spreading Aedes mosquito-borne viral infectious diseases. Recently in Bangladesh it has emerged as an important public health issue. Chikungunya virus (CHIKV) mostly spread by Aedes aegypti and Aedes albopictus, an anthropophilic mosquito species widely distributed in Asia, Europe, Africa and America. Our objective was to determine the clinical, biochemical and radiological features of patients at the acute phase of CHIKV infection. The purpose of study was to evaluate the literature and summarize the current state of CHIKV-associated disease, including clinical presentation, diagnosis, risk factors for development of severe diseases, complications and treatment.

We present 253 confirmed cases of chikungunya having different clinical presentations occurring among adult patients from different background including foreigner in Dhaka city, admitted in a tertiary level hospital situated in Gulshan from march'17 to November 2017 . All patients

\section{Introduction:}

Chikungunya is caused by a vector-borne virus of genous Alphavirus and family Togavirus. Aedes aegypti and Aedes albopictus are the usual vectors, which are also responsible for transmission of dengue, hence there is concurrence or co-incidences of dengue and chikungunya in endemic regions ${ }^{1}$. In Bangladesh, over the past two decades, dengue had been recognized as endemic disease with high incidence during the rainy

a. Dr. Safia Binte Rabbani, Specialist in Medicine, United Hospital Limited.

b. Dr. Pradip Ranjan Saha, Consultant, Department of Internal Medicine, United Hospital Ltd.

c. Dr. Md. Iqbal Hossain, Consultant, Department of Internal Medicine, United Hospital Ltd.

d. Dr. Afsana Begum, Consultant, Department of Internal Medicine, United Hospital Ltd.

e. Dr. Md. Jahangir Talukder, Consultant, Department of Internal Medicine, United Hospital Ltd.

Address of Correspondence: Dr. Safia Binte Rabbani, Specialist, Medicine Department, United Hospital Limited. Phone: 01715003919 , Email:safia_rabbani@yahoo.com

Received: 10 June, 2018

Accepted: 16 March, 2019 had fever and joint pain. Other common features were rash, diarrhoea, vomiting, confusion, and altered liver biochemistry. Adult patients with multiple co-morbidities admitted in hospital with male preponderance of $59 \%$ and rest were female $41 \%$. Most common complication was post CHIKV arthritis (79\%) and rest of the less common complications were post viral asthenia (34\%), myocarditis (27\%), pneumonitis (30\%). Dengue was excluded in all patients. Paracetamol remained the mainstay of treatment during febrile periods, but around $62 \%$ of the patients had prolonged joint symptoms requiring non-steroidal antiinflammatory drugs, colchicines, steroid. Among joint involvement, ankle joints were commonly involved joint presented with post viral arthritis. Since there is no specific treatment of chikungunya, prevention through vector control and public health education is the key.

Key words: Chikungunya fever, Viral infectious disease.

(J Bangladesh Coll Phys Surg 2019; 37: 124-129)

DOI: https://doi.org/10.3329/jbcps.v37i3.41734

seasons. Last year's outbreak of chikungunya also parallels the same seasonal and environmental characteristics of dengue in Dhaka, Bangladesh. Aedes albopictus $^{2}$ was the main vector in 2011 outbreak in Dhaka. Scientists forecasted chikungunya as an emerging viral infection in Bangladesh in 2014 as well ${ }^{3}$.

\section{Methods :}

A retrospective observational study enrolled 253 consecutive patients older than 20 years with fever, arthralgia, rash admitted at the tertiary level hospital situated in gulshan, Dhaka city from March 2017 to November 2017. Biological confirmation of CHIKV infection was obtained for all patients, either by detection of CHIK virus RNA in the blood during acute stage by RT-PCR or the presence of anti-CHIK virus specific immunoglobulin $\mathrm{M}$ by ELISA depending on delayed presentation. And dengue was excluded by doing either dengue NS1 on initial presentation or ICT for dengue in late presentation. But those patients who had co-infection of dengue and CHIKV were included in the study. Three groups were defined: first group of 80 viremic patients (positive CHIKV RT-PCR), second 
group of 173 patients with acute post-viremic infection (positive anti-CHIKV IgM and negative IgG) and last group of 5 patients having both Dengue and chikungunya ( dengue NS1 +ve, Ig M for CHIKV positive or RTPCR positive for CHIKV). All statistical data were analyzed by SPSS 22 software package. P value $<0.05$ considered as significant.

\section{Results:}

The population was predominantly male $(59 \%)$ and the median age was 55 years ranging from $20-70$ years. Highest recorded number patients were admitted between june' 17 to September '17. Patients with CHIKV viremia presented typically with asymmetrical bilateral polyarthralgia $(96.5 \%)$ affecting the lower limbs (98\%) and small joints of hands $(74.8 \%)$, as well as asthenia $(88.6 \%)$, headache (70\%), nausea,vomiting (63.3\%), myalgia (59\%), exanthems (47.8\%). Vertigo, cutaneous dysesthesia, pharyngitis and haemorrhages were seldom observed. Pruritis was less frequent during the viremic than post-viremic phase $(13.9 \%$ vs. $41.2 \%$; $<<0.001)$, whereas lymphopenia was more frequent $(87.6 \%$ vs. $39.4 \% ; \mathrm{p}<0.001)$. Others biological abnormalities included leukopenia (38.3\%), thrombocytopenia (37.3\%), increased AST and ALT blood levels (31.6 and $37.3 \%$, respectively). Age under 50 were associated with a benign course of the disease.

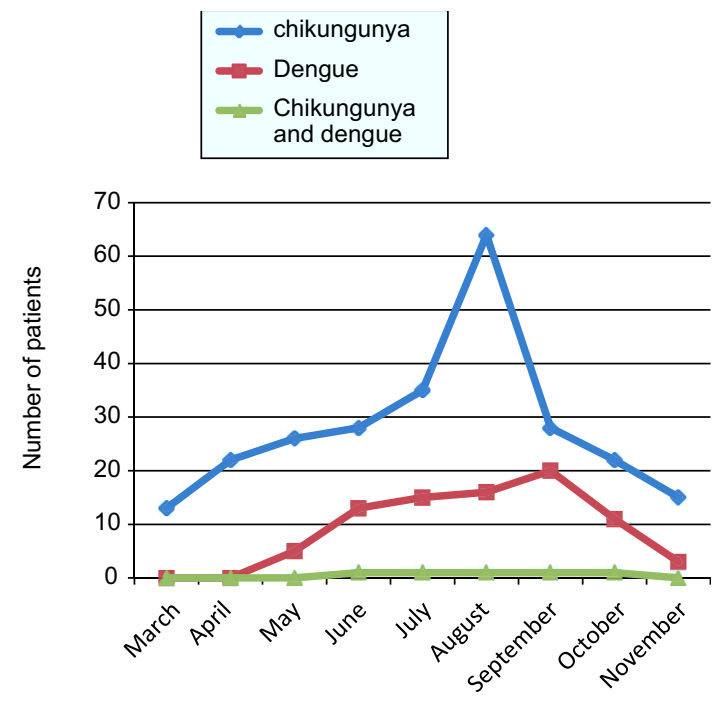

Fig.-1: Month distribution of chikungunya and dengue fever 2017

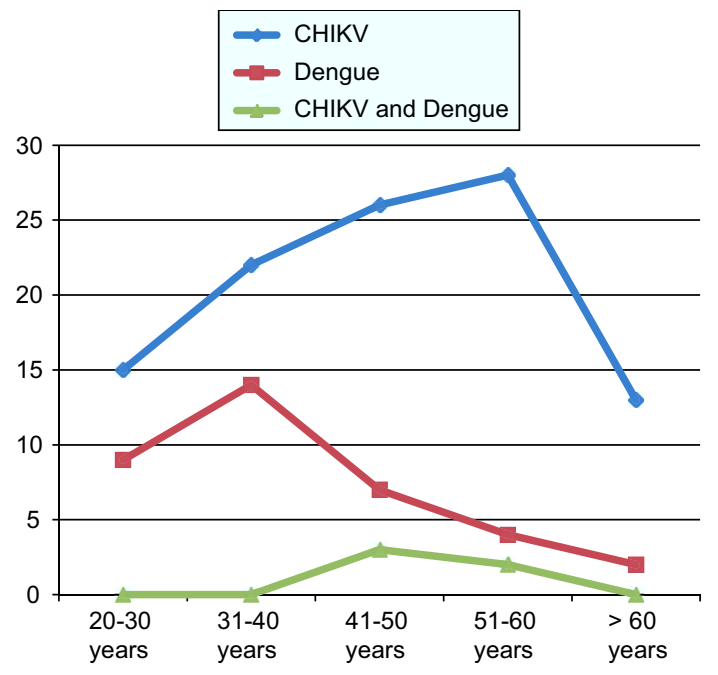

Fig.-2: Age distribution of patients

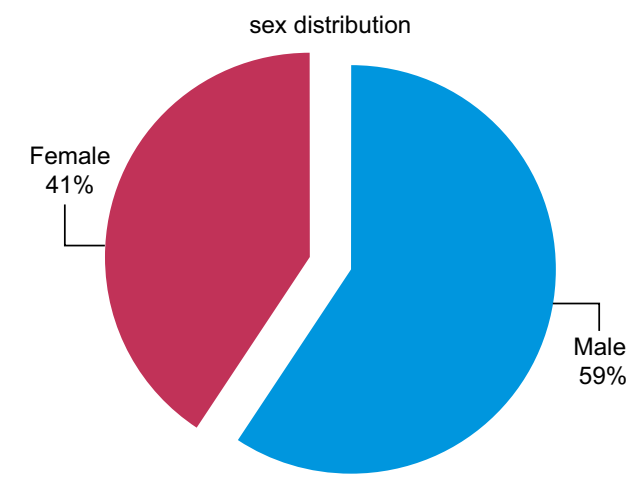

Fig.-3: sex distribution in patients of CHIKV

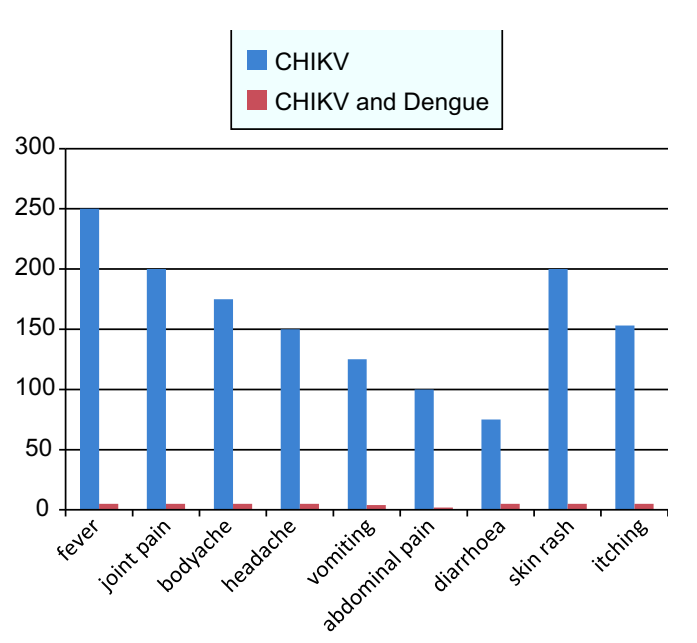

Fig.-4: symptoms of patients 
Table-I

Shows complication of CHIKV, Dengue fever

\begin{tabular}{lcccc} 
& CHIKVn=248 & Denguen=40 & CHIKV \& Denguen=5 & P-value \\
\hline Post viral asthenia & $85(34 \%)$ & $5(6 \%)$ & $2(40 \%)$ & $\mathrm{P}<0.01$ \\
Post viral Arthritis & $200(79 \%)$ & 0 & $1(20 \%)$ & \\
Hyperpigmentation & $2(0.7 \%)$ & 0 & 0 & \\
Encephalitis & $3(1 \%)$ & $3(3.6 \%)$ & 0 & \\
Myocarditis & $72(27 \%)$ & $8(9.6 \%)$ & 0 & \\
Heart failure & $6(2 \%)$ & 0 & 0 & \\
Pneumonitis & $76(30 \%)$ & 0 & 0 & \\
Pneumonia & $3(1.1 \%)$ & 0 & 0 & \\
Acute kidney injury & 0 & 0 & $3(60 \%)$ & \\
Bleeding manifestation & 0 & $20(43 \%)$ & &
\end{tabular}

\section{Table-II}

Joint Involvement in Chikungunya fever

\begin{tabular}{lcc}
\multicolumn{3}{c}{ Joint Involvement in Chikungunya fever } \\
& CHIKV n=248, $<<0.05$ & $\begin{array}{c}\text { CHIKV \& Dengue co infection } \\
\mathrm{n}=5, \mathrm{p}<0.001\end{array}$ \\
\hline Knee joint & 47 & 2 \\
Ankle joint & 153 & 3 \\
Meta-tarsal joint & 80 & 0 \\
Hip joint & 5 & 0 \\
Shoulder joint & 5 & 0 \\
Elbow joint & 3 & 0 \\
Wrist joint & 30 & 0 \\
Meta-carpophalangeal & 3 & 0 \\
Inter-phalangeal joint & 5 & 0 \\
\hline
\end{tabular}

In the rainy season, the highest recorded patients were admitted in hospital from June to September (Fig-1) 2017. Among them the rate of middle aged patients admission were common (Fig-2) due to their multiple co-morbidities like ischemic heart disease, hypertension, diabetes, osteoarthritis. During hospital stay few patients developed complication related to cardio-respiratory system but subsequent follow up in OPD revealed that most of them i.e 79\% patients having post viral arthritis and $34 \%$ patient having asthenia. (Table-1). In table 1 there were also showed comparison of complication of CHIKV with dengue patients who were then admitted in hospital. Our study also showed ankle joint involvement was most commonly involved in CHIKV infection. (Table-2)

\section{Discussion :}

Chikungunya virus (CHIKV) is a mosquito-borne alphavirus that circulates predominantly in tropical and subtropical regions, potentially affecting over 1 billion people. "Chikungunya" is a Makonde word in Tanzania meaning "to walk bent over" or "that which bends up" 4 .

One of the most consistent and debilitating manifestations of CHIKV-induced disease in people consists of an often widespread, severe and incapacitating arthralgia, occurring in $85-100 \%$ of people with symptomatic infection $5,6,7$. In fact, this disease manifestation is so common in CHIKVinduced disease, it has been reported that the presence of concurrent high fever and arthralgia has a specificity of $99.6 \%$ and positive predictive value of $84.6 \%$ for 
the diagnosis of CHIKV infection ${ }^{8}$. Recent outbreak in Bangladesh, CHIKV infection results in severe fever, rash and debilitating polyarthralgia lasting weeks to months. In most cases these symptoms resolves in approximately 2 weeks ${ }^{9}$. However, as many as $88 \%$ of people can have arthralgia persisting for 1 month after the initial symptoms ${ }^{10,11}$. In our study, $79 \%$ patients having post viral arthalgia which is consistent with this study.

Arthralgia associated with CHIKV infection is often considered to be the result of tenosynovitis and enthesopathy ${ }^{12}$ and can be associated with paresthesia of the overlying skin ${ }^{13}$. In most cases of CHIKV infection, patients develop symmetrical oligo or polyarthralgia following the initial onset of fever ${ }^{14}$. While any joint can be affected, those that are most commonly reported are the distal extremities such as wrists, metacarpal and interphalangeal joints, as well as the ankles and meta-tarsophalangeal joints, though some studies have indicated that the knee is also commonly affected joint ${ }^{10,12,15}$. But, in our study most commonly affected joints were ankle joint, knee joint, wrist joint. (Table-2)

Sebastian study shows, in the acute stages of the disease, results of a complete blood cell count can be variable, though leukopenia, often as a result of lymphopenia, is one of the most common presentations ${ }^{16}$. This will often persist for the first week of infection, and is occasionally accompanied by thrombocytopenia and an increased hematocrit. Our study population were also consistent with this result and in most cases these parameters returned to normal in the second week of infection. Though a one case developed persistent pancytopenia who required blood transfusion and GM-CSF injection. In our study, blood chemistry results were often normal throughout the disease course, though increased liver enzymes including ALT and AST have been reported.

Bouquillard study shows , $42.9 \%$ of patients had normal radiographs at the time of diagnosis of chronic CHIKV arthritis, while $57.1 \%$ had evidence of joint space narrowing and $23.8 \%$ had visible erosions. However, at the follow-up 24 months later, only $19 \%$ of patients had normal radiographs, and $81 \%$ of patients demonstrate joint space narrowing and erosions which increased than previous ${ }^{17}$. In our study, $95 \%$ patients having normal radiograph of joints while $5 \%$ having change of age related osteoarthritis in knee and ankle joints at time of diagnosis.

Patients with chikungunya fever may present cardiovascular complication including myocarditis and heart failure. Simon et al, ${ }^{18}$ they demonstrated clinical, biological and morphological evidence of myocarditis and serological documented CHIKV infection. In our study, Viral myocarditis mostly diagnosed clinically in $27 \%$ patients having chest pain, palpitation, breathlessness and on examination there were irregular pulse, bradycardia or tachycardia, ECG evidence of sinus bradycardia, ventricular premature contraction (VPC) or atrial fibrillation, ST depression. Laboratory evidence were raised high sensitive troponin I, raised brain natriuretic peptide (BNP) and echocardiogram evidence of hypokinesia of left ventricle or arrhythmia noted. No myocardial biopsy were taken as it was not feseable. Our study is relatively consistent with Gonzalez Carta et al. ${ }^{19}$ Their study shows arrhythmia occurred in $45 \%$ cases, among them $33 \%$ were bradycardia and others were VPC, tachy-arrhythmia. Serial ECG evaluation remains best guide for the prognosis. ${ }^{20}$

A wide array of skin and mucus membrane lesion have been documented in literature by Indian worker during their epidemic of diseases. ${ }^{21,22}$ In their study ,hyperpigmentation in chikungunya fever may be of different types including centro facial, diffuse pigmentation of face, extrimities and pigmentation of existing acne lesion. In our study, two patients developed hyperpigmentation. One male patient had diffuse pigmentation of whole body and in another female patient developed hyperpigmentation in extensor surface of forearm. Female patient's rash worsened into violaceous, vasculitic lesion over the extrimities and subsequently clinically diagnosed as a case of post chikungunya vasculitis. CHIKV can be a rare cause of vasculitis.

Currently, specific treatment modalities for acute and chronic CHIKV-induced disease do not exist, and the majority of treatment plans consist of supportive and symptomatic care. In acute stages of the disease, this consists predominantly of rest, antipyretics and analgesics ${ }^{11}$. In our study, most of the patients were managed with paracetamol, cold compression of joints 
according to national guideline published by IEDCR (Institute of Epidemiology, Disease control and research). 158 patients $(62.45 \%)$ required NSAIDs. Patients of chronic kidney disease were managed with colchicine and few required short term oral steroid. We also observed that bed rest was the main stay of treatment. Patients who were admitted in early phase of the diseases got early recovery due to adequate bed rest.

NSAIDs can have variable effects in chronic cases. In one study in Bangladesh, 2 of 6 patients treated acutely with NSAIDs had persistent pain at 2-3 months ${ }^{9}$. In some cases joint pain may resolve, but stiffness remains ${ }^{23}$, perhaps suggesting permanent alterations in articular or peri-articular tissues resulting in decreased mobility. It should also be noted that aspirin is not recommended due to bleeding risk ${ }^{24}$.

Simon et al study ${ }^{25}$, refractory to NSAID treatment cases, short-term courses of corticosteroids have been attempted with variable success. While corticosteroid treatments are usually not recommended due to the potential for a severe rebound of arthritis and tenosynovitis after therapy is ended, in severe cases of inflammatory polyarthritis refractory to NSAID treatment, the benefits may outweigh the risks. In Waymouth study cases, steroids helped resolve symptoms of arthralgia, tenosynovitis, entrapment syndrome and Raynaud's syndrome ${ }^{8}$, while in Foissac cases, they had no effect ${ }^{14}$.

The efficacy of specific antiviral medications are also unknown, though in vitro studies have demonstrated potential utility of ribavirin against alphaviruses in Burt F.J study ${ }^{26}$. In a small cohort of ten patients, decreased pain and swelling were reported in most patients 27. However, larger scale, controlled efficacy studies have not been performed to assess the true clinical utility of ribavirin in treating CHIKV-induced disease. In our study, we did not treat any patients with anti viral therapy.

While there have been millions of people affected by CHIKV within the last decade, methods of prevention and treatment are still lacking. Preventive measures focus on the mosquito vector and include recommendations such as wearing long sleeves, making use of mosquito nets, elimination of mosquito breeding sites and use of insecticides. Several vaccines are in various stages of development, though none are yet commercially available. ${ }^{28}$

\section{Conclusion:}

Persistent and disabling arthralgia was a frequent concern of patients who had experienced severe chikungunya virus infection. Treatments are generally symptomatic and supportive, and the efficacies of potentially more specific therapies are currently unknown .Further long term studies are needed to evaluate the prevalence of persistent arthralgia in the general population to determine the real burden of the disease.

\section{Acknowledgement:}

To the Department of hematology and biochemistry lab, United hospital Limited.

\section{Reference:}

1. Furuya-Kanamori L, Liang S, Milinovich G, Soares Magalhaes $\mathrm{RJ}, \mathrm{Clements} \mathrm{AC}, \mathrm{Hu} \mathrm{W}$, et al. Co-distribution and co-infection of chikungunya and dengue viruses. BMC Infect Dis. 2016;3(16):84. doi: 10.1186/s12879-016-1417-2.

2. First identified outbreak of chikungunya in Bangladesh, 2008. Health Sci Bull. 2009;7:1-6.

3. Hassan R, Rahman MM, Moniruzzaman M, Rahim A, Barua S, Biswas $\mathrm{R}$, et al. Chikungunya-an emerging infection in Bangladesh: a case series. J Med Case Rep. 2014;8:67. doi: 10.1186/1752-1947-8-67.

4. Chopra A., Anuradha V., Lagoo-Joshi V., Kunjir V., Salvi S., Saluja M. Chikungunya virus aches and pains: an emerging challenge. Arthritis Rheum. 2008;58(9):2921-2922. doi: 10.1002/art.23753.

5. Higgs S., Ziegler S.A.A nonhuman primate model of chikungunya disease. J. Clin. Invest. 2010;120(3):657-660. doi: 10.1172/ JCI42392.

6. Sissoko D., Malvy D., Ezzedine K., Renault P., Moscetti F., Ledrans M., Pierre V. Post-epidemic Chikungunya disease on Reunion Island: course of rheumatic manifestations and associated factors over a 15 -month period. PLoS Negl. Trop. Dis. 2009;3(3):e389. doi: 10.1371/journal.pntd.0000389.

7. Schilte C., Staikowsky F., Couderc T., Madec Y., Carpentier F., Kassab S., Albert M.L., Lecuit M., Michault A. Chikungunya virus-associated long-term arthralgia: a 36-month prospective longitudinal study. PLoS Negl. Trop. Dis.2013;7(3):e2137.doi:10.1371/journal.pntd.0002137.

8. Waymouth H.E., Zoutman D.E., Towheed T.E. Chikungunyarelated arthritis: case report and review of the literature. Semin. Arthritis Rheum. 2013;43(2):273-278. doi: 10.1016/ j.semarthrit.2013.03.003.

9. Khatun S, Chakraborty A, Rahman M, Banu N, Rahman MM, Hasan SMM, et al. An outbreak of chikungunya in rural 
Bangladesh, 2011. PLoS Negl Trop Dis. 2015;9(7):e0003907. doi: 10.1371/journal.pntd.0003907.

10. Ali Ou Alla S., Combe B. Arthritis after infection with Chikungunya virus. Best Pract. Res. Clin. Rheumatol. 2011;25(3):337-346. doi: 10.1016/j.berh.2011.03.005.

11. Calabrese L.H. Emerging viral infections and arthritis: the role of the rheumatologist. Nat. Clin. Pract. Rheumatol. 2008;4(1):2-3.

12. Malvy D., Ezzedine K., Mamani-Matsuda M., Autran B., Tolou H., Receveur M.C., Pistone T., Rambert J., Moynet D., Mossalayi D. Destructive arthritis in a patient with chikungunya virus infection with persistent specific IgM antibodies. BMC Infect. Dis. 2009;9:200.

13. Jaffar-Bandjee MC, Das T, Hoarau JJ, Krejbich Trotot P, Denizot $\mathrm{M}$, Ribera A, et al. Chikungunya virus takes centre stage in virally induced arthritis: possible cellular and molecular mechanisms to pathogenesis. Microbes Infect/Inst Pasteur. 2009;11(14-15):1206-18.

14. Foissac M., Javelle E., Ray S., Guérin B., Simon F. PostChikungunya rheumatoid arthritis, Saint Martin. Emerg. Infect. Dis. 2015;21(3):530-532.

15. Chow A., Her Z., Ong E.K., Chen J.M., Dimatatac F., Kwek D.J., Barkham T., Yang H., Rénia L., Leo Y.S., Ng L.F. Persistent arthralgia induced by Chikungunya virus infection is associated with interleukin-6 and granulocyte macrophage colonystimulating factor. J. Infect. Dis. 2011;203(2):149-157.

16. Sebastian M.R., Lodha R., Kabra S.K. Chikungunya infection in children. Indian J. Pediatr. 2009;76(2):185-189.

17. Bouquillard E., Combe B. A report of 21 cases of rheumatoid arthritis following Chikungunya fever. A mean follow-up of two years. Joint Bone Spine. 2009;76(6):654-657.

18. Simon F, Paule P, Oliver M : chikungunya induced myopericarditis , towards an increase in DCM in counties with epidemic. Am J Trop Med Hyg. 2008; 78(2):212-3

19. K.A Gonzalez Carta, Ivan J Mendoza, Vicenta Finizola et al Bradycardia as a manifestation of chikungunya myocarditis. Circulation .2018;134:A13292
20. Villamil GomezWE, Ramirez-VallejoE, Catdona-Ospire JA et al . Electrocardiographic alteration in patients with chikungunya fever from sucre, Colombia: a 42 case series. Travel Med Infect Dis ,2016;14(5): 510-2.

21. Inamader AC, Palit A, Sampagavi VV, Raghunath S, Deshmukh NS, Cuteneous manifestation of CHIKV. Observation made during a recent outbreak in south india. Int J Dermatol 2008; $47: 154-9$.

22. Debabrata Bandyopadhyay, Sudip kumar ghosh . Mucocuteneous manifestation of Chikungunya fever. Indian J Dermatol. 2010 Jan-Mar; 55(1):64-67.

23. Volpe A., Caramaschi P., Angheben A., Marchetta A., Monteiro G., Bambara L.M., Bisoffi Z. Chikungunya outbreakremember the arthropathy. Rheumatology (Oxford) 2006;45(11):1449-1450.

24. Thiberville S.D., Moyen N., Dupuis-Maguiraga L., Nougairede A., Gould E.A., Roques P., de Lamballerie X. Chikungunya fever: epidemiology, clinical syndrome, pathogenesis and therapy. Antiviral Res. 2013;99(3):345-370.

25. Simon F., Javelle E., Cabie A., Bouquillard E., Troisgros O., Gentile G., Leparc-Goffart I.,Hoen B., Gandjbakhch F., Rene-Corail P., Franco J.M., Caumes E., Combe B., Poiraudeau S., Gane-Troplent F., Djossou F., Schaerverbeke T., Criquet-Hayot A., Carrere P., Malvy D., Gaillard P., Wendling D.French guidelines for the management of chikungunya (acute and persistent presentations). November 2014. Med. Mal. Infect. 2015;45(7):243-263.

26. Burt F.J., Rolph M.S., Rulli N.E., Mahalingam S., Heise M.T. Chikungunya: a re-emerging virus. Lancet. 2012;379(9816): 662-671.

27. Ravichandran R., Manian M. Ribavirin therapy for Chikungunya arthritis. J. Infect. Dev. Ctries. 2008;2(2):140-142.

28. Ahola T., Couderc T., Ng L.F., Hallengärd D., Powers A., Lecuit M., Esteban M., Merits A., Roques P., Liljeström P. Therapeutics and vaccines against chikungunya virus. Vector Borne Zoonotic Dis. 2015;15(4):250-257. 